\title{
AGUDIZACIONES ENDODONTICAS: “FLARE-UPS” (PARTE I)
}

\author{
Olga Lucía Torres Borrero \\ Odontóloga Universidad Santo Tomás, Estudiante de II año, Especialización en Endodoncia, \\ Universidad Santo Tomás.
}

Dirección de correo electrónico: olgatorresborrero@yahoo.com.mx

\begin{abstract}
RESUMEN
La agudización endodóntica se caracteriza por el desarrollo de dolor, edema o ambos, posterior a una intervención endodóntica. Generalmente, los factores causantes son: daño mecánico, químico y/o microbiano a la pulpa o tejidos perirradiculares. De todos ellos, los microorganismos son indiscutiblemente el mayor agente etiológico de las agudizaciones. Se presentan varios regímenes de tratamiento para el alivio del dolor durante la terapia endodóntica que incluyen el alivio de la oclusión, premedicación, drenaje y medicaciones sistémicas y dentro del conducto radicular. El objetivo de este primer artículo fue realizar una revisión de la literatura acerca de su definición, incidencia y posibles factores desencadenantes. En la segunda parte se tratará la etiopatogenia y la adopción de medidas preventivas apropiadas para reducir significativamente la incidencia de este indeseable fenómeno clínico. [Torres OL. Agudizaciones endodónticas: "Flare ups" (Parte I). Revista Ustasalud Odontología 2007; 6: 123 - 130]
\end{abstract}

Palabras clave: Agudización, Tratamiento endodóntico, Infección del conducto radicular.

\section{ENDODONTIC FLARE UPS: PART I}

\begin{abstract}
Inter-appointment flare-ups are characterized by the development of pain, swelling or both, following an endodontic treatment. The etiologic factors of flare-ups included mechanical and/or microbial injury to the pulp or perirradicular tissues. Of these factors, microorganisms are probable the major causative agents of flare-ups. Various treatment regimens for the relief of pain during endodontic therapy, including relief of drainage, and intracanal and systemic medications are presented. The aim of this first paper was to make a literature review about its definition, incidence and relative factors that may cause flare ups. The second paper will review the etiology and some preventive aids to reduce its incidence.
\end{abstract}

Key words: Flare ups, Endodontic treatment, Root canal infection.

Recibido para publicación: 10 de julio de 2007. Aceptado para publicación: 10 de diciembre de 2007.

\section{INTRODUCCIÓN}

La realización de un tratamiento endodóntico es un procedimiento que, por lo general, despierta muchas "expectativas" en el paciente, más aún si éste presenta sintomatología. Posiblemente, se deba a la creencia popular de que los tratamientos de conductos son dolorosos. Aunque uno de sus objetivos es proporcionar alivio al paciente, puede llegar a ser frustrante tanto para el paciente como para el odontólogo, que el tratamiento efectuado ocasione dolor o inflamación; algunas veces llega a ser sorpresiva una sintomatología dolorosa postoperatoria, si el paciente previo a la realización del tratamiento, se encontraba asintomático.
La sintomatología que puede presentar un paciente durante la realización de un tratamiento endodóntico se conoce como agudización endodóntica. Es una complicación que se presenta de manera inesperada durante la terapia endodóntica, sorprende tanto al paciente como al clínico tratante y requiere su pronta atención. De ahí, surge la inquietud por conocer, entre otras cosas, su incidencia y las causas que la producen para manejarla de manera adecuada y prevenir cuanto más sea posible.

Se ha reportado gran variación en la frecuencia de flare-ups; parece ser que ocurre en un promedio del 14 al $16 \%$. Ciertos factores influyen significativa- 
mente en el desarrollo de flare-ups entre cita y cita, entre éstos se encuentra la edad, el género, el tipo de diente, el estado pulpar, la presencia de dolor preoperatorio, algunas alergias y la presencia de un tracto sinuoso. ${ }^{1}$

Los factores causales de flare-ups entre citas comprenden daño mecánico y químico a la pulpa, y a los tejidos perirradiculares. De hecho, muchos casos de flare-ups ocurren como un resultado de una inflamación perirradicular aguda (periodontitis apical aguda supurativa o absceso apical agudo). Independiente del tipo de lesión, la intensidad de la respuesta inflamatoria es directamente proporcional al tejido dañado. ${ }^{2}$

El objetivo de esta primera revisión sobre agudizaciones endodónticas es discutir las diferentes definiciones sobre el tema, su incidencia y los factores que pueden estar relacionados con esta entidad.

\section{AGUDIZACIÓN ENDODÓNTICA}

\section{Definiciones:}

Este tipo de dolor postoperatorio tiende a ser un tanto confuso, incluso en su denominación; así se puede encontrar que se le denomina agudización endodóntica, exacerbación aguda o "flare-ups" (en inglés).

A través de los años, diversos autores han estudiado el fenómeno de las agudizaciones endodónticas y han elaborado diferentes definiciones: Bartels y colaboradores (1968) la definen como "una condición de dolor o inflamación que resulta en una consulta de emergencia". ${ }^{3,4}$ Seltzer y Naidorf (1985) la señalan como un problema que se presenta durante o después de la terapia endodóntica, desarrolla dolor e inflamación. La misma puede ocurrir posterior al acceso del conducto, aún sin haberlo instrumentado, y debido a la magnitud de la sintomatología puede alarmar tanto al paciente como al clínico tratante. ${ }^{3}$ Naidorf (1985) se refirió a las agudizaciones endodónticas como "una parte del precio al hacer endodoncia, no importa que tan cuidadoso se sea en su realización". ${ }^{3}$

Goldman y colaboradores, en 1988, la describen como "una situación que se presenta en un paciente asintomático después de la preparación biomecánica completa del o los conductos, que resulta en dolor severo y constante y/o edema dentro de 1 ó 2 días siguientes". ${ }^{5}$ Matusow (1986) define el fenómeno como la exacerbación, manifestada como una celulitis aguda que ocurre periódicamente durante el tratamiento endodóntico inicial de dientes no vitales, asintomáticos y que usualmente se encuentran intactos asociados a lesiones periapicales. ${ }^{6}$

Se ha considerado que la agudización está relacionada con una infección y se ha definido como una consulta de emergencia no programada, necesaria debido a la presencia de dolor e inflamación combinadas o solamente inflamación. ${ }^{7}$ Trope (1990) la define como "la manifestación clínica de una inflamación periapical aguda comúnmente asociada con infección".

Walton y Fouad (1992) afirman que la agudización endodóntica puede presentarse dentro de las primeras horas o pocos días después de iniciado el tratamiento endodóntico, el paciente presenta dolor o inflamación o la combinación de ambas. ${ }^{4}$ El problema puede ser lo suficientemente severo como para interrumpir el estilo de vida del paciente, y éste haga contacto con el odontólogo, para requerir una consulta de emergencia y un tratamiento inmediato. Torabinejad y Walton (1991), la describen como "fenómenos reactivos a los mediadores químicos y a la presión, creados como resultado de las reacciones inflamatorias en los tejidos perirradiculares". ${ }^{9}$

Cada autor varía en cierta medida el concepto de una agudización endodóntica de acuerdo con la etiología y los signos y síntomas, esta situación hace difícil comparar los resultados de los estudios. Rimmer (1993) afirma que ninguna de esas definiciones describe todos los parámetros que conciernen a la agudización endodóntica como un todo. ${ }^{10}$ Estas diferencias han llevado a dicho autor a sugerir un índice para utilizar un lenguaje común que permita a la comunidad odontológica medir y estudiar de una manera completa este fenómeno. En la presente revisión bibliográfica, la definición de agudización que se va a utilizar es la siguiente: la agudización endodóntica es una complicación que se presenta durante la realización de la terapia endodóntica de dientes no vitales asociados con imágenes radiolúcidas apicales, que puedan o no encontrarse asintomáticos y que, una vez, iniciado el tratamiento endodóntico requieren de una consulta de urgencia, a consecuencia del dolor severo y/o la inflamación que se presenta. 
No se considerará agudización al dolor posterior a la instrumentación que ocurre en los dientes vitales como consecuencia de una respuesta inflamatoria aguda debido a la pulpectomía y subsiguiente preparación del conducto, ya que esta molestia postoperatoria generalmente no es severa y es una respuesta normal al tratamiento. ${ }^{11}$

\subsection{FACTORES DESENCADENANTES:}

Se les ha dividido en tres importantes grupos:

- Irritantes del conducto radicular: se encuentran bacterias, subproductos bacterianos, tejido pulpar necrótico, así como sustancias nocivas $\mathrm{y}$ antígenos que penetran al conducto y tejidos periapicales. $^{4}$

- Factores terapéuticos iatrogénicos: la sobre o subinstrumentación, las soluciones irrigadoras, los medicamentos colocados dentro del conducto, la hiperoclusión y una debridación incompleta. ${ }^{4}$

- Factores del huésped: se encuentran la edad, el género, la presencia de dolor preoperatorio, el tamaño de la lesión apical, el tratamiento por segunda intención y la presencia de fístula. ${ }^{4}$

\subsection{INCIDENCIA:}

La frecuencia con la que se presentan las agudizaciones endodónticas es variable, esta variación es el resultado parcial del análisis de diferentes factores y condiciones con las que se han relacionado las agudizaciones endodónticas. Por ejemplo, Barnett y Tronstad (1989) realizaron un estudio retrospectivo, en el que utilizaron pacientes asintomáticos, con pulpas necróticas asociadas a lesiones periapicales, en donde consideraron como agudización la presencia de dolor y/o inflamación. ${ }^{12}$ Estos autores determinaron una incidencia de aproximadamente un $5.5 \%$. Otros han reportado distintos porcentajes de incidencia de agudizaciones, de acuerdo con los factores considerados por los mismos.

Walton y Fouad (1992) consideran que el problema con muchos de estos estudios es que han sido realizados de manera retrospectiva, sin controles, han tenido un número reducido de pacientes o han manejado variables no definidas. Dichos autores realizaron un estudio prospectivo, en un período de cuatro meses, y evaluaron 946 citas de pacientes que se encontraban bajo tratamiento endodóntico; obtuvieron como resultado una incidencia de $3.17 \%{ }^{4}$
1.3.1 Factores relacionados con la incidencia de agudizaciones

Los factores que se han relacionado con un mayor número de agudizaciones son:

\section{- Edad y género del paciente.}

Algunos autores sugieren que las agudizaciones se presentan con mayor frecuencia en personas menores de 50 años. ${ }^{7,13}$ Parece que la posible razón de la discrepancia en las edades se relaciona con la anatomía del conducto radicular y con los cambios dentinarios que en él ocurren. Con los años hay un incremento de las calcificaciones pulpares, una disminución del diámetro de los conductos radiculares y un incremento en la calcificación de los túbulos dentinarios. Por lo tanto, los conductos en las personas mayores son estrechos y contienen una menor cantidad de restos necróticos e infectados lo que conduce a una menor probabilidad para que ocurra una agudización endodóntica. 7,13 Walton y Fouad consideran que no hay relación entre la edad y la incidencia de este fenómeno. ${ }^{4}$

Balaban y colaboradores (1984) consideran que no hay relación entre el género y la incidencia de agudizaciones. ${ }^{13}$ Otros autores consideran que las agudizaciones se presentan con mayor frecuencia en el sexo femenino que en el masculino. ${ }^{14-16}$ Por otra parte, autores como Walton y Fouad (1992) no encontraron diferencias significativas relacionadas con el género aunque si establecieron que se presentan en una proporción de 2:1 (femenino:masculino). ${ }^{4}$

\section{- Diente involucrado.}

Se ha relacionado una mayor incidencia de agudizaciones en los incisivos laterales superiores y en los premolares inferiores. ${ }^{7}$ Balaban y colaboradores consideraron que las agudizaciones se presentan con mayor frecuencia en los incisivos laterales superiores, con una incidencia de $38.1 \%$ y $45.4 \%$ respectivamente. ${ }^{13}$ Estos autores suponen que la razón de esta alta incidencia sea la curvatura distal y palatina que presentan estos dientes; si en esta porción apical quedan restos que no han sido preparados adecuadamente, las bacterias allí presentes podrán multiplicarse y producir sustancias inflamatorias. ${ }^{?}$ 
Naidorf (1985) sugirió que los molares inferiores son propensos a producir una agudización debido a que, entre las raíces mesiales se encuentra una anatomía radicular difícil de limpiar. ${ }^{17}$ De igual manera, Genet y colaboradores afirmaron que el dolor postoperatorio depende del número de conductos presentes, incrementándose cuando existen varios conductos. ${ }^{16}$ No obstante Walton y Fouad (1992) no encontraron diferencias significativas entre grupos de dientes ni entre las arcadas (superior e inferior). ${ }^{4}$

\section{- Historia de dolor preoperatorio.}

Genet y colaboradores realizaron un estudio en 443 dientes, a fin de determinar los factores preoperatorios y operatorios asociados con dolor después de la primera cita; obtuvieron como resultado que el dolor postoperatorio se presentó en un $27 \%$ de los casos, de este porcentaje, $5 \%$ presentó dolor severo y $22 \%$ dolor moderado, relacionaron el dolor postoperatorio con la presencia de varios factores. ${ }^{16}$

Uno de estos factores fue la presencia de dolor preoperatorio; estos autores afirmaron que los casos de pulpas no vitales con dolor preoperatorio presente al momento de la cita inicial, tienen más probabilidades de presentar dolor postoperatorio que los casos de pulpas vitales, independientemente de que éstas presenten o no, dolor preoperatorio. Las probabilidades disminuyen en los casos de pulpas necróticas asintomáticas. Estos hallazgos han sido reportados con anterioridad por otros autores. . $^{11,14,18-21}$

Así mismo, Walton y Fouad encontraron que el factor más importante relacionado con la incidencia de agudizaciones, fue la presencia de dolor preoperatorio severo, el cual resultó en un $19 \%$ de incidencia. ${ }^{4}$

\section{- Presencia y tamaño de la lesión periapical.}

La presencia de lesión periapical ha sido relacionada con la incidencia de agudizaciones. Si bien autores como Fox y colaboradores encontraron una disminución en la frecuencia de dolor postoperatorio en aquellos casos que presentaban imagen radiolúcida apical, ${ }^{14}$ otros autores como Sundqvist, Yoshida y colaboradores, y Walton y Fouad afirmaron que las agudizaciones endodónticas se presentan, con mayor frecuencia, en dientes con patología periapical. ${ }^{4,14,22,23}$ Por su parte, Trope sugirió que las agudizaciones no ocurren en dientes sin signos radiográficos de lesión periapical. ${ }^{8}$

En cuanto al tamaño de la lesión periapical, varios autores coinciden en que los dientes con grandes zonas de rarefacción apical, son más propensos a sufrir de exacerbaciones dolorosas, que aquellos con lesiones de tamaño reducido. Genet y colaboradores (1987) encontraron que el dolor postoperatorio se presentó cuando la imagen radiolúcida fue mayor de $5 \mathrm{~mm}$ de diámetro. ${ }^{11}$

\section{- Inflamación preoperatoria.}

Walton y Fouad (1992) la encontraron como segundo factor en importancia, localizada o difusa, con una incidencia de un $15 \%$ de los casos estudiados. $^{4}$

\section{- Repetición del tratamiento endodóntico.}

En los últimos años se ha señalado una mayor incidencia de agudizaciones en dientes que requieren la repetición del tratamiento endodóntico. Trope (1990) realizó un estudio con un total de 474 dientes, de los cuales 147 eran retratamientos. Sus resultados en la incidencia de agudizaciones fueron de $3.79 \%$ para dientes que no presentaban tratamiento previo, y $4.08 \%$ para los casos de retratamiento. ${ }^{8}$

En un estudio posterior, el mismo autor realizó los tratamientos en una sola cita y obtuvo una incidencia de agudizaciones de un $13.6 \%$ en los casos de retratamiento. ${ }^{24}$ Este autor sugirió que al tratar de retirar la gutapercha contenida dentro del conducto, ésta tiende a extruir las bacterias y detritus hacia el periápice, donde causarán inflamación aguda y dolor severo. Así mismo afirmó que el solvente utilizado puede contribuir con un incremento en la respuesta inflamatoria.

Por otra parte, Walton y Fouad (1992) también reportaron una mayor incidencia de agudizaciones en los casos de retratamiento, aunque no encontraron una mayor incidencia entre los mismos y los casos de tratamientos convencionales. ${ }^{4}$

\section{- Número de citas.}

Mulhern y colaboradores (1982) realizaron un estudio sobre la incidencia de dolor postoperatorio 
después de un tratamiento endodóntico realizado en una sola cita en 60 dientes monorradiculares, asintomáticos y con necrosis pulpar, tratados por dos estudiantes de posgrado. Treinta dientes fueron tratados en tres citas, se evaluaron clínica y radiográficamente. Un cuestionario subjetivo les fue entregado a los pacientes a fin de registrar las experiencias de dolor. No encontraron diferencias significativas en la incidencia de dolor postoperatorio entre el grupo al que se les realizó el tratamiento endodóntico en varias citas y el que fue tratado en una sola cita. ${ }^{15}$

Así mismo, en el estudio de Trope en 1991, todos los dientes fueron instrumentados a una medida predeterminada e irrigados con hipoclorito de sodio al $0.5 \%$. Los conductos fueron obturados sin registrar la presencia o ausencia de síntomas ni el diagnóstico de la condición apical. A los pacientes se les entregaron instrucciones postoperatorias por escrito y una prescripción de 600 mg de ibuprofeno para tomarlo en caso de dolor moderado a intenso. Si se presentaba un dolor severo o inflamación, el paciente debía llamar inmediatamente y será considerado como una agudización. ${ }^{24}$

De acuerdo con los hallazgos de este estudio, el autor concluyó que los dientes sin lesión apical no se agudizan y pueden ser tratados en una sola cita. Los dientes con lesión apical sin tratamiento previo, pueden ser tratados en una sola cita con una baja probabilidad de que ocurra una agudización endodóntica. Las agudizaciones fueron mayores en los dientes con periodontitis apical que necesitaron retratamiento por lo que los retratamientos en una sola cita no son aconsejables, en estos casos. ${ }^{24}$

Eleazer y Eleazer (1998) realizaron un análisis retrospectivo con un grupo numeroso de tratamientos endodónticos en molares con pulpas necróticas. Dichos dientes fueron tratados por un mismo operador, utilizaron siempre la misma técnica de preparación biomecánica de los conductos radiculares, y se trataron unos en una sola sesión y otros en sesiones múltiples. Los autores concluyeron que no existía diferencia significativa entre los tratamientos realizados en una sola sesión y los realizados en varias sesiones con respecto a la presencia de agudizaciones endodónticas. ${ }^{25}$

La Tabla 1 resume algunos de los estudios realizados a través del tiempo en la incidencia de dolor postoperatorio y agudizaciones en una sola sesión.

Tabla 1. Incidencia de dolor postoperatorio y agudizaciones en los casos realizados en una sesión.

\begin{tabular}{lccccc}
\hline \multicolumn{1}{c}{ Investigador } & Año & No. de casos & Condición pulpar & Ninguno o ligero & Moderado o severo \\
\hline Ferranti & 1959 & 340 & Necrótica & $91.0 \%$ & $9.0 \%$ \\
Fox y colaboradores & 1970 & 270 & Vital-Necrótica & $90.0 \%$ & $10.0 \%$ \\
O`keefe & 1976 & 55 & Vital-Necrótica & $98.0 \%$ & $2.0 \%$ \\
Soltanoff & 1978 & 282 & Vital-Necrótica & $81.0 \%$ & $19.0 \%$ \\
Ashkenaz & 1979 & 359 & Vital & $96.0 \%$ & $4.0 \%$ \\
Rudner \& Oliet & 1981 & 98 & Vital-Necrótica & $88.5 \%$ & $11.5 \%$ \\
Mulhern y colaboradores & 1982 & 30 & Necrótica & $76.5 \%$ & $23.5 \%$ \\
Oliet & 1983 & 382 & Vital-Necrótica & $89.0 \%$ & $11.0 \%$ \\
Roane y colaboradores & 1983 & 359 & Vital-Necrótica & $85.0 \%$ & $15.0 \%$ \\
Alacam & 1985 & 212 & Vital & $86.0 \%$ & $14.0 \%$ \\
Morse y colaboradores & 1986 & 200 & Necrótica & $98.5 \%$ & $1.5 \%$ \\
Morse y colaboradores & 1987 & 106 & Necrótica & $93.4 \%$ & $6.6 \%$ \\
Abbott y colaboradores & 1988 & 195 & Necrótico & $97.4 \%$ & $2.6 \%$ \\
& 1989 & 60 & Vital & $97.0 \%$ & $3.0 \%$ \\
Fava & 1991 & 120 & Necrótico & $95.0 \%$ & $5.0 \%$ \\
& 1994 & 90 & Vital & $94.0 \%$ & $6.0 \%$ \\
Trope & 1995 & 60 & Necrótico & $94.0 \%$ & $6.0 \%$ \\
Walton y Fouad & 1991 & 226 & & $98.2 \%$ & $1.8 \%$ \\
Abbott & 1992 & 935 & Vital-Necrótico & $97.4 \%$ & $2.6 \%$ \\
\hline
\end{tabular}

Tomado de Cohen \& Burns, Pathways of the Pulp, 7th Ed., 1998 


\section{- Sobreinstrumentación.}

Ha existido una gran controversia acerca del límite apical hasta el que debe llegar la instrumentación de los conductos radiculares cuando los dientes presentan lesiones periapicales. Grossman afirmó que, idealmente, una urgencia endodóntica no debería ocurrir durante el tratamiento. Sin embargo, si ésta ocurre ocasionalmente, es probable que sea el resultado de una instrumentación vigorosa que ha transportado microorganismos, restos dentinarios o ambos, a través del foramen apical. ${ }^{26}$

La sobreinstrumentación como causa de la incidencia de agudizaciones ha sido reportada por Balaban y colaboradores (1984). Estos autores consideraron que, posiblemente la instrumentación a través del ápice sea la única razón por la cual los dientes, que habían sido instrumentados completamente en la primera cita, se hayan exacerbado, debido a una respuesta inflamatoria aguda desencadenada por la instrumentación dentro de los tejidos periapicales. ${ }^{13}$

Seltzer (1979) estableció que cuando los tejidos periapicales son maltratados por instrumentos manipulados a través del ápice de un diente infectado, provocan gran cantidad de daño tisular. Los tejidos periapicales traumatizados sirven como nido para el crecimiento y multiplicación de los microorganismos. ${ }^{27}$

Se debe tener en cuenta que existen estudios en los que se ha determinado que la sobreinstrumentación de los conductos radiculares infectados produce bacteremias transitorias. Bender y colaboradores encontraron que hubo un $31 \%$ de bacteriemias en los dientes en los cuales se realizó una sobreinstrumentación; al cabo de $10 \mathrm{mi}$ nutos la incidencia de bacteriemias descendió a $0 \%{ }^{28}$ En contraste, Baumgartner y colaboradores sugirieron que los procedimientos endodónticos no quirúrgicos producen una baja incidencia de bacteriemias (3\%). ${ }^{29}$

Wittgow y Sabiston (1975) afirmaron que durante la sobreinstrumentación, la aparición de microorganismos anaerobios dentro del hueso alveolar puede ser un factor desencadenante de una agudización endodóntica. ${ }^{30}$ Sin embargo, Sundqvist (1976) sugirió que una combinación específica de bacterias en la pulpa podría ser más importante para la inducción de las exacerbaciones que la forma en la que se realiza la instrumentación. ${ }^{22}$ Por otra parte, Balaban y colaboradores encontraron que cuando la instrumentación del conducto era corta, la incidencia de agudizaciones resultó en un $14.3 \%$ de los casos. ${ }^{13}$ No obstante, si la instrumentación se realizaba hasta el límite del ápice radiográfico, las agudizaciones disminuían a un $10 \%$ de los casos.

Morse y colaboradores en la evaluación comparativa de tres de sus estudios afirmaron que en el primero de ellos (1986), la instrumentación de los conductos se realizó hasta el límite del ápice radiográfico y la incidencia de agudizaciones fue de $19.6 \%$. En el segundo, se realizó una instrumentación vigorosa aproximadamente hasta el centro de la lesión periapical, y se prescribió antibiótico a los pacientes que debían tomar al observar el primer signo de inflamación; la incidencia de agudizaciones fue de $4.3 \%$. En el tercer estudio, se usaron antibióticos profilácticamente, los tratamientos fueron realizados en una sola cita y la instrumentación se realizó de la misma forma que en el estudio anterior; la incidencia de agudizaciones fue de $1.5 \%$. Posteriormente, en 1987, realizaron un estudio prospectivo para comparar la sobreinstrumentación con la instrumentación dentro del conducto, en casos de dientes asintomáticos con lesiones periapicales, no encontraron diferencias significativas. ${ }^{7,31}$

\section{- Historia de alergia sistémica.}

Algunos autores afirman que los pacientes alérgicos son propensos a sufrir agudizaciones endodónticas. Goldman y colaboradores (1988) realizaron un estudio restrospectivo en 1260 pacientes tratados consecutivamente, en un período de 25 meses. Los resultados de este estudio mostraron que los pacientes con historia de alergia sistémica fueron tres veces más propensos a sufrir una agudización que aquellos pacientes sin historia de alergia. Estos autores sugirieron que se debe a que en los pacientes alérgicos los niveles de inmunoglobulina $\mathrm{E}$ (IgE) son mayores que en los pacientes no alérgicos y que cuando un antígeno del conducto radicular es impulsado hacia los tejidos periapicales, se podría desencadenar una reacción de hipersensibilidad inmediata o Tipo I. ${ }^{32}$ 
Svetcov y colaboradores (1983) encontraron niveles elevados de IgE y anaerobios obligados en pacientes que presentaron agudizaciones durante la realización del tratamiento endodóntico. ${ }^{33}$ Este hallazgo ha sido apoyado por otros autores y será analizado profundamente en la parte II de esta revisión. ${ }^{34-36}$

\section{- Enfermedades sistémicas.}

Con relación a este punto, autores como Morse y colaboradores no encontraron diferencias significativas entre los pacientes que presentaban alguna enfermedad sistémica y los pacientes aparentemente sanos, en cuanto a una predisposición por parte de los primeros a sufrir de agudizaciones endodónticas; mencionaron que, probablemente, el tamaño de muestra no era lo suficientemente grande como para llegar a una conclusión con respecto a la influencia de las mismas. ${ }^{31}$

Sin embargo, Abbott y colaboradores (1988) en un estudio prospectivo realizado al azar, con la finalidad de medir la eficacia de la profilaxis antibiótica en dientes asintomáticos con necrosis pulpar y asociados a una patología periapical, encontraron que solo dos pacientes se agudizaron y que éstos presentaban dentro de su historia el antecedente médico de diabetes mellitus insulino-dependiente. ${ }^{7}$ Consideraron que, probablemente, en estos pacientes se encuentre alterada la capacidad para defenderse de las infecciones, por lo que recomendaron duplicar la dosis de antibióticos aplicados de manera profiláctica a fin de controlar esta complicación.

\section{- Técnica de instrumentación.}

Otro factor considerado en la incidencia de dolor postoperatorio en dientes con pulpas necróticas es la extrusión de material a través del foramen apical. Fairbourn y colaboradores (1987) realizaron un estudio in vitro para comparar cuatro técnicas de instrumentación, con la finalidad de recolectar y pesar la cantidad de restos extruídos a través del foramen apical. Basados en un análisis de varianza, las técnicas fueron clasificadas de menor a mayor cantidad de restos extruídos de la siguiente manera: técnica sónica, técnica de ensanchamiento cervical (cervical flaring techni$q u e)$, técnica ultrasónica y técnica de paso atrás (step-back technique). ${ }^{37}$
En el mismo año, Ruiz-Hubard y colaboradores compararon la técnica de paso atrás con la técnica de corona-abajo (crown-down pressureless technique) para conocer la cantidad de restos extruídos apicalmente. Sus resultados mostraron que ninguna técnica es efectiva en prevenir la extrusión de restos a través del foramen apical, pero sí encontraron que fue mayor la cantidad de los mismos cuando se utilizó la técnica de paso atrás. ${ }^{38}$

Basados en estos estudios, autores como Fava y Trope utilizan en sus estudios técnicos el ensanchamiento cervical, a fin de disminuir la posibilidad de extruir restos o detritus que puedan desencadenar una agudización endodóntica, y obtenido en sus tratamientos una baja incidencia de agudizaciones. ${ }^{24,39}$

\section{BIBLIOGRAFÍA}

1. Siquiera JF. Microbial causes of endodontic flare-ups. Int Endod J 2003; 36: 453 - 463.

2. Seltzer S, Naidorf IJ. Flare-ups in endodontics: I Etiological factors. J Endod 2004; 30: 476 - 481.

3. Seltzer S, Naidorf IJ. Flare-ups in endodontics: II. Therapeutic measures. J Endod 2004; 30: 482 - 484.

4. Richard W, Fouad A. Endodontic interappoiment flareups: A prospective study of incidence and related factors. J Endod 1992; 18: 172 - 177.

5. Goldman M, Rankin C, Mehlman R, Santa C. The immunologic implications and clinical management of the endodontic flare-up. Compend Contin Educ Dent 1988; 10: $126-130$.

6. Matusow RJ. Acute pulpal-alveolar cellulitis syndrome IV. Exacerbations during endodontic treatment: A clinical study of specific microbial isolates and their etiologic role. Part 1. Oral Surg Oral Med Oral Pathol 1986; 61: $90-95$.

7. Abbott A, Koren L, Morse D, Sinai I, Doo R, Furst MA. Prospective randomized trial on efficacy of antibiotic prophylaxis in asymptomatic teeth with pulpal necrosis and associated periapical pathosis. Oral Surg Oral Med Oral Pathol 1988; 66: 722 - 733.

8. Trope M. Relationship of intracanal medicaments to endodontic flare-ups. Endod Dent Traumatol 1990; 6: $226-229$.

9. Torabinejad M, Walton RE. Urgencias endodónticas. En: Walton RE, Torabinejad M. Endodoncia Principios y Práctica Clínica. Ed. Interamericana. McGraw-Hill. México. 1991; p. 311 - 315. 
10. Rimmer A. The flare-up index: a quantitative method to describe the phenomenon. J Endod 1993; 19: 255 256.

11. Genet JM, Wesselink PR, Thoden van Velze SK. The incidence of preoperative and postoperative pain in endodontic therapy. Int Endod J 1986; 19: 221 - 229.

12. Barnett F, Tronstad L. The incidence of flare-ups following endodontic treatment. J Dent Res 1989; 68 (special issue): 1253.

13. Balaban FS, Skidmore AE, Griffin JA. Acute exacerbations following initial treatment of necrotic pulp. J Endod 1984; 10: $78-81$

14. Fox J, Atkinson JS, Dinin AP. Incidence of pain following one-visit endodontic treatment. Oral Surg Oral Med Oral Pathol 1970; 30: $123-130$.

15. Mulhern JM, Patterson SS, Newton CW, Ringel AM. Incidence of postoperative pain after one-appointment endodontic treatment of asymptomatic pulpal necrosis in single-rooted teeth J Endod 1982; 8: 370 - 375.

16. Genet JM, Hart AAM, Wesselink PR, Thoden van Velzen SK. Preoperative and operative factors associated with pain after the first endodontic visit. Int Endod J 1987; 20: $53-64$.

17. Naidorf IJ. Endodontic flare-ups: bacteriological and immunological mechanisms. J Endod 1985; 11: 462 - 464.

18. Seltzer S, Bender IB, Ehrenreich J. Incidence and duration of pain following endodontic therapy. Oral Surg Oral Med Oral Pathol 1961; 14: $74-82$.

19. Clem WH. Posttreatment endodontic pain. J Am Dent Assoc 1970; 81: 1166 - 1170.

20. O'Keefe EM. Pain in endodontic therapy: preliminary study. J Endod 1976; 2: 315 - 319.

21. Maddox DL, Walton RE, Davis CO. Incidence of posttreatment endodontic pain related to medicaments and other factors. J Endod 1977; 3: 447.

22. Sundqvist G. Bacteriological studies of necrotic dental pulps. Umeå University Odontological Dissertations $\mathrm{N}^{\circ}$ 7. University of Umeå, Sweden. 1976: 1 - 94.

23. Yoshida M, Fukushima H, Yamamoto K, Ogawa K, Toda $\mathrm{T}$, Sagawa, H. Correlation between clinical symptoms and microorganisms isolated from root canals of teeth with periapical pathosis. J Endod 1987; 13: 24 - 28.

24. Trope M. Flare-ups rate of single-visit endodontics. Int Endod J 1991; 24: 24 - 26.

25. Eleazer, P.; Eleazer, K. Flare up rate in pulpally necrotic molars in one-visit versus two-visit endodontic treatment. J Endod 1998; 24: $614-616$.

26. Grossman LI. Endodontic emergencies. Oral Surg Oral Med Oral Pathol 1977; 43: 948 - 953.

27. Seltzer S. Endodoncia Consideraciones biológicas de los procedimientos endodónticos Ed. Mundi. Argentina. 1979; p. 199 - 345

28. Bender IB, Seltzer S, Tashman S, Meloff G. Dental procedures in patients with rheumatic heart disease. Oral Surg Oral Med Oral Pathol 1963; 16: 446.
29. Baumgartner JC, Heggers JP, Harrison JW. Incidence of bacteremias related to endodontic procedures. II. Surgical endodontics. J Endod 1977; 3: 399 - 402.

30. Wittgow W, Sabiston C. Microorganisms from pulpal chambers of intact teeth with necrotic pulps. J Endod 1975; $1: 168-171$.

31. Morse DR, Furst ML, Belott RM, Lefkowitz RD, Spritzer IB, Sideman BH. A prospective randomized trial comparing periapical instrumentation to intracanal instrumentation in cases of asymptomatic pulpal - periapical lesions. Oral Surg Oral Med Oral Pathol 1987; 64: 734 $-741$.

32. Goldman M, Rankin C, Mehlman R, Santa C. The inmunologic implications and clinical management of the endodontic flare-up. Compendium 1988; 10: 126 - 130.

33. Svetcov SD, DeAngelo JE, McNamara T, Nevins AJ. Serum immunoglobulin levels and bacterial flora in subjects with acute oro-facial swellings. J Endod 1983; 9: $233-235$

34. Nevins A, Levine S, Gayer Y, Svetcov S. Sensitization via IgE-mediated mechanism in patients with chronic periapical lesions. J Endod 1985; 11: 228 - 230.

35. Nevins A, Friedman L, DeVita R, Schacter W. Local injection of Benadryl for the prevention of iatrogenic endodontic flare-ups. Endod Dent Traumatol 1988; 4: $90-91$.

36. Nevins A, Verhelle R, Feldman M, Berman D. Local prophylactic Benadryl injections in an attempt to reduce postinstrumental pain. J Endod 1994; 20: 296 - 298.

37. Fairbourn DR, McWalter GM, Montgomery S. The effect of four preparation techniques on the amount of apically extruded debris. J Endod 1987; 13: 102 -108.

38. Ruiz-Hubard EE, Gutmann JL, Wagner MJ. A quantitative assessment of canal debris forced periapically during root canal instrumentation using two different techniques. J Endod 1987; 13: 554 - 558.

39. Fava LR. A comparison of one versus two appointment endodontic therapy in teeth with non-vital pulps. Int Endod J 1989; 22: 179 - 183. 